\title{
Una propuesta de clasificación taxonómica del problema de recogida de productos defectuosos
}

\section{A taxonomical classification proposal for product recalls}

\author{
Juan M. Cogollo-Flórez ${ }^{1 \mathrm{a}}$, Mariana Restrepo-Hincapié ${ }^{1 \mathrm{~b}}$ \\ ${ }^{1}$ Departamento de Calidad y Producción, Instituto Tecnológico Metropolitano - ITM, Colombia. \\ Correo electrónico: ${ }^{\mathrm{a}}$ juancogollo@itm.edu.co, ${ }^{\mathrm{b}}$ marianarestrepo256115@ correo.itm.edu.co \\ Orcid: ${ }^{\mathrm{a}}$ 0000-0002-6101-3134, ${ }^{\mathrm{b}} 0000-0001-9150-0585$
}

Recibido: 24 octubre, 2020. Aceptado: 2 febrero, 2021. Versión final: 12 mayo, 2021.

\section{Resumen}

El estudio de la problemática de retiro de productos defectuosos del mercado (recalls) es un tema relevante para la gestión de la calidad y la gestión de cadenas de suministro. En este artículo se identifican los principales aportes, enfoques y metodologías propuestos en la literatura científica para tratar el tema de la recogida de productos defectuosos en el mercado, a través de un análisis bibliométrico y la clasificación taxonómica de los aportes realizados en los últimos 20 años. Para ello, se realizó una revisión de literatura en las bases de datos Taylor and Francis, EBSCO, Science Direct, Scopus, Springer Link, IEEE, Google Scholar y ProQuest. El análisis bibliométrico permitió identificar los principales clústeres en relación con la problemática. La clasificación taxonómica permitió agrupar los aportes en seis categorías: tipo de aporte, enfoque de calidad, enfoque del problema, tipo de metodología, características de los datos y categoría de producto. Se evidenció que los sectores con mayor nivel de ocurrencia de retiros de productos son la industria de juguetes, el sector automotor, suministros eléctricos y la industria alimenticia.

Palabras clave: análisis bibliométrico; control de la calidad; clasificación taxonómica; gestión de cadenas de suministro; gestión de la calidad; productos inseguros; productos defectuosos; protección del consumidor; recogida de productos; revisión de literatura.

\begin{abstract}
The Product Recall study is a relevant issue for both quality management and supply chain management. This paper identifies the main contributions, approaches, and methodologies proposed in the scientific literature to address product recall through a bibliometric analysis and a taxonomical classification regarding works made over the last 20 years. For this, a literature review was carried out in the Taylor and Francis, EBSCO, Science Direct, Scopus, Springer Link, IEEE, Google Scholar, and ProQuest databases. The bibliometric analysis allowed us to identify the main clusters related to product recalls. The taxonomical classification allowed the grouping of works into six categories: type of work, quality approach, problem approach, type of methodology, data characteristics, and product category. The sectors with the highest level of occurrence of product recalls are the toy industry, automotive sector, electrical supplies, and food industry.
\end{abstract}

Keywords: bibliometric analysis; quality control; taxonomical classification; supply chain management; quality management; unsafe product; defective product; consumer protection; product recall; literature review.

ISSN impreso: 1657 - 4583. ISSN en línea: 2145 - 8456, CC BY-ND 4.0 두요 (1) @

Como citar: J. M. Cogollo-Flórez, M. Restrepo-Hincapié, "Una propuesta de clasificación taxonómica del problema de recogida de productos defectuosos," Rev. UIS Ing., vol. 20, no. 3, pp. 111-120, 2021, doi: 10.18273/revuin.v20n3$\underline{2021007}$ 


\section{Introducción}

Los retiros de productos del mercado (recalls) se deben a la presencia de defectos o fallas que desencadenan un riesgo de daño sustancial o potencial para los consumidores, como resultado del incumplimiento de la legislación o de las normas de seguridad, uso incorrecto de materiales, mal etiquetado, entre otras causas [1]. La gestión de los recalls se centra en la recogida de todos los productos involucrados que se encuentren en proceso de producción, en puntos de venta o en poder de los consumidores [2]. La problemática de los recalls no es propia de un sector industrial o jurisdicción específica. Se han presentado casos en diversos sectores, tales como la industria alimenticia, automotriz, juguetes, equipos eléctricos, productos farmacéuticos, alimentos para animales, entre otras [2], [3], [4]. De igual forma, es frecuente su ocurrencia en países de diferentes continentes y diferente grado de desarrollo económico o industrial [5].

Los productos inseguros o defectuosos en el mercado siguen siendo frecuentes, a pesar de las prácticas de control y verificación de la calidad que regularmente implementan las empresas [6], [7], [8], [9]. Son una señal de alerta de un malfuncionamiento en la coordinación de la calidad de una organización con sus socios en la parte ascendente o descendente de la cadena de suministro [10], [11]. La integración de la calidad en la gestión de la cadena de suministro para evitar recalls implica que cada miembro participe activamente en las actividades de diseño, elaboración y distribución de los productos [12], [13].

Además del enfoque académico, se han desarrollado varias iniciativas gubernamentales para tratar el problema de los recalls a través del registro de la información sobre su ocurrencia [14]. Entre ellas se destaca la iniciativa Global recalls de la Organización para la Cooperación y Desarrollo Económico (OECD).

Global Recalls reúne datos e información de los retiros de productos emitidos por los países miembros de la OECD, con el fin de identificar la predominancia del problema a partir de las diferentes categorías y sectores de la industria.

El portal de la $\mathrm{OECD}^{1}$, cuenta con 36 categorías que han sido clasificadas según el tipo de producto y frecuencia de retiro. La página web permite desplegar todas las categorías de las cuales se desea obtener información: fecha de retiro, nombre del producto en cuestión, jurisdicción de destitución, país donde se fabricó el producto, idioma y el respectivo enlace de las diferentes autoridades competentes $\mathrm{o}$ entes reguladores, relacionados con cada país donde se ve reflejado el retiro originalmente.

Algunos países como Australia, Francia, Alemania, Portugal, Reino Unido, Estados Unidos, Canadá, Vietnam y España se han registrado en el portal, consolidando información sobre fabricantes, tipo de producto, identificación del lote, tipo de defecto, número de artículos involucrados, entre otras características.

Dada la importancia de los recalls en el contexto de la gestión de la calidad y la gestión de cadenas de suministro, el objetivo de este artículo es identificar los principales aportes, enfoques y metodologías propuestos en la literatura científica para tratar el tema, a través de un análisis bibliométrico y clasificación taxonómica de los aportes realizados en los últimos 20 años. La organización del artículo es la siguiente: en la sección II se describe la metodología utilizada, en la sección III se muestran los resultados del análisis bibliométrico y la clasificación taxonómica desarrollada y, se finaliza, con las conclusiones en la sección IV.

\section{Metodología}

La metodología utilizada en la investigación consistió en cinco etapas: 1) formulación de pregunta de investigación, 2) determinación de criterios de búsqueda, 3) determinación de criterios de inclusión y exclusión, 4) búsqueda en bases de datos y 5) clasificación taxonómica de los resultados.

\subsection{Formulación de pregunta de investigación}

Con el fin de estudiar las diferentes tendencias y perspectivas acerca de la problemática latente del retiro de productos en diversos sectores de la industria, se formuló la siguiente pregunta de investigación: ¿Es posible realizar una clasificación de los enfoques metodológicos que se han utilizado durante los últimos 20 años para tratar el problema de los recalls?

\subsection{Criterios de búsqueda}

La búsqueda se llevó a cabo comprendiendo un periodo desde el año 2000 hasta el 2020 y usando palabras clave de la temática bajo estudio a través de las siguientes ecuaciones de búsqueda específicas: "Product recalls" AND "methodology", "Product recalls" AND "quality management", "Product recalls" AND "supply chain management", "Product recalls" AND "quality" AND

${ }^{1}$ https://globalrecalls.oecd.org 
"supply chain", "Product recalls" AND "legislation", "Product recalls" AND "prevention", "Product recalls" AND "announcements", "Product recalls" AND "traceability", "Product recalls" AND "risk management", "Product recalls" AND "safety" AND "security" y "Product recalls" AND "trends" AND "technology".

\subsection{Criterios de inclusión y exclusión}

Para la selección de los documentos pertinentes a los objetivos de la investigación, se determinaron dos criterios de inclusión: primero, si el documento presenta un enfoque legislativo-gubernamental o académico para el estudio, gestión o prevención de recalls, y, segundo, si el documento presenta un análisis de recalls, abordando aspectos de casos específicos.

Similarmente, se descartaron aquellos artículos que cumplían al menos uno de los siguientes criterios de exclusión:

1) El documento trata el tema de recalls, pero no describe alguna metodología, variables o indicadores relacionados.

2) El documento trata el tema de recalls, en un área diferente a la gestión de calidad en la cadena de suministro. En la siguiente sección se presentan los resultados de las dos últimas etapas de la metodología: la búsqueda en bases de datos y el análisis de resultados.

\section{Resultados y discusión}

\subsection{Resultados de búsqueda en bases de datos}

En la tabla 1 se muestran los resultados del número de artículos recuperados en las bases de datos seleccionadas aplicando las ecuaciones de búsqueda mencionadas anteriormente.

Se evidencia que las bases de datos ProQuest, Google Scholar y Science Direct arrojaron un mayor número de resultados en relación con el tema y sus palabras clave asociadas.

También, en la figura 1 se muestra el resultado del análisis bibliométrico usando el software Vosviewer, a partir de las palabras clave más utilizadas por los autores, identificados por medio de clústeres de colores. Se nota que predomina en el clúster de color rojo la palabra clave "supply chain management", la cual cuenta con 96 palabras enlazadas entre sí, 185 palabras que tienen mayor afinidad y 149 ocurrencias que se visualizan en el mapa.

En el clúster de color verde se encuentra la palabra clave "quality improvement" con 117 palabras enlazadas entre sí, 231 palabras que se identificaron con mayor afinidad y 165 ocurrencias dentro del mapa. En el clúster de color morado aparece la palabra clave "supply chain", que cuenta con 47 palabras enlazadas entre sí, 62 palabras con mayor afinidad y 51 ocurrencias dentro del mapa.

Tabla 1. Número de artículos recuperados en búsqueda inicial

\begin{tabular}{|c|c|c|c|c|c|c|c|c|}
\hline Palabras clave & 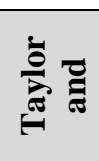 & 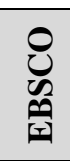 & 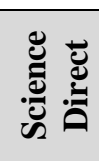 & 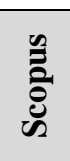 & 离 & 空 & 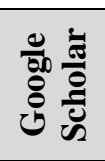 & 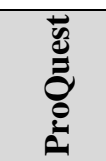 \\
\hline "Product recalls" AND "methodology" & 184 & 23 & 1090 & 78 & 875 & 0 & 7630 & 8410 \\
\hline "Product recalls" AND "quality management" & 70 & 27 & 422 & 44 & 311 & 4 & 2920 & 2400 \\
\hline "Product recalls" AND "supply chain management" & 91 & 46 & 320 & 40 & 255 & 5 & 3130 & 2338 \\
\hline $\begin{array}{c}\text { "Product recalls" AND "quality" AND "supply } \\
\text { chain" }\end{array}$ & 133 & 40 & 801 & 48 & 573 & 2 & 5920 & 9567 \\
\hline "Product recalls" AND "legislation" & 77 & 143 & 700 & 84 & 516 & 0 & 5580 & 11861 \\
\hline "Product recalls" AND "prevention" & 144 & 143 & 924 & 84 & 582 & 2 & 6770 & 11443 \\
\hline "Product recalls" AND "announcements" & 46 & 23 & 263 & 38 & 249 & 0 & 2680 & 4718 \\
\hline "Product recalls" AND "traceability" & 55 & 41 & 1073 & 79 & 294 & 7 & 2760 & 2255 \\
\hline "Product recalls" AND "risk management" & 67 & 50 & 450 & 47 & 375 & 2 & 4020 & 5689 \\
\hline "Product recalls" AND "safety" AND "security" & 90 & 50 & 554 & 10 & 557 & 1 & 7680 & 10895 \\
\hline "Product recalls" AND "trends" AND "technology" & 89 & 45 & 975 & 7 & 682 & 1 & 6470 & 11449 \\
\hline Total & 1046 & 631 & 7572 & 559 & 5269 & 24 & 55560 & 81025 \\
\hline
\end{tabular}

Fuente: elaboración propia. 


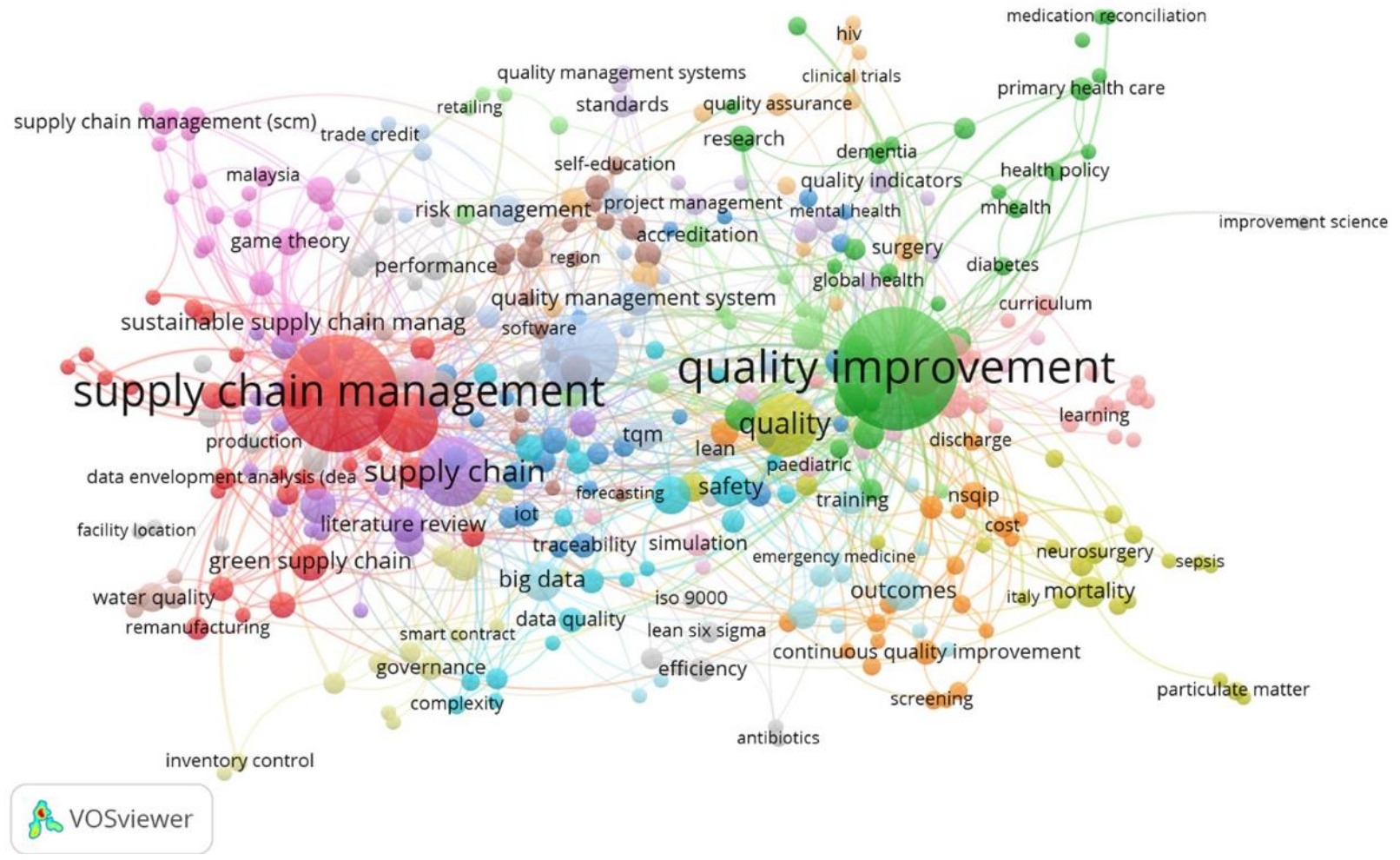

Figura 1. Mapa bibliométrico de resultados. Fuente: elaboración propia usando VOSviewer.

Finalmente, en el clúster de color amarillo aparece la palabra clave "quality", que cuenta con 53 palabras enlazadas entre sí, 76 palabras con mayor afinidad y 42 ocurrencias dentro del mapa. Todas estas de gran importancia, de constante repetición y colaboración entre la búsqueda de los artículos, ya que evidencian la interrelación entre la gestión de la calidad y la gestión de cadenas de suministro para el estudio de los recalls.

\subsection{Clasificación taxonómica de estudios sobre recalls}

La clasificación taxonómica desarrollada es la respuesta a la pregunta de investigación inicialmente planteada, acerca de la posibilidad de realizar una clasificación de los enfoques metodológicos que se han propuesto en la literatura durante los últimos 20 años para tratar el problema de los recalls.

Usando las 11 palabras clave mencionadas anteriormente y realizando la búsqueda en las bases de datos seleccionadas, se escogieron inicialmente un total de 477 artículos. Luego, aplicando los criterios de inclusión y exclusión, se seleccionaron finalmente 57 documentos, los cuales fueron analizados para desarrollar la clasificación taxonómica mostrada en la tabla 2, usando un enfoque jerárquico de dos niveles [15].
El primer nivel comprende las siguientes categorías: 1) tipo de aporte, 2) enfoque de calidad, 3) enfoque del problema, 4) tipo de metodología, 5) características de los datos y 6) categoría de producto. Luego, para el segundo nivel, los artículos son caracterizados al interior de cada categoría en una o varias de las subcategorías presentadas.

La primera categoría, tipo de aporte, comprende la clasificación fundamental de cada artículo en teórico o práctico. La segunda categoría, enfoque de calidad, hace referencia al enfoque intraorganizacional tradicional o al enfoque de calidad en cadenas de suministro (supply chain quality management, SCQM). La tercera categoría, enfoque del problema, se refiere a la perspectiva académica, práctica o legal. La cuarta categoría, tipo de metodología, se refiere al enfoque cualitativo o cuantitativo del aporte. La quinta categoría, características de los datos, se refiere al uso de datos reales o sintéticos o el no uso de datos. La última categoría, categoría de producto, comprende las diferentes tipologías de productos analizados en los trabajos propuestos.

El cumplimiento de los atributos de cada subcategoría se marca con una $\mathrm{X}$ en las celdas respectivas. En la tabla 2 se observa que no hay subcategorías marcadas tres veces 
o menos y que el $100 \%$ de las subcategorías están marcadas cuatro o más veces, lo que permite afirmar que la taxonomía desarrollada es robusta para clasificar concisamente los artículos seleccionados sobre estudios de la problemática de recalls.

Según el tipo de aporte, se encuentra que el $74 \%$ de los aportes presentan un enfoque teórico sobre la problemática de recalls basados en antecedentes, evolución y opciones de tratamiento. El $28 \%$ presenta un enfoque aplicado utilizando principalmente modelos de optimización estocástica. Según el enfoque de calidad, el $19 \%$ de los trabajos presentan un enfoque tradicional de calidad intraorganizacional, basado en que la responsabilidad de la calidad de los productos recae sobre cómo se gestiona la toma de decisiones en el interior de las organizaciones, teniendo como pilar fundamental los costos de prevención, evaluación, fallas internas y fallas externas. El $61 \%$ presenta un enfoque a la gestión de la calidad de cadenas de suministro, estableciendo que la mayoría de los productos inseguros o defectuosos ocurren por fallas en la gestión de alguna etapa de la cadena. El $25 \%$ presenta otro tipo de enfoques, como el análisis de juicios del producto e intenciones de comportamiento en un contexto de retiro de productos, considerando la percepción del consumidor, la cultura y la responsabilidad social empresarial.

Según el enfoque del problema, el $58 \%$ presenta un enfoque relacionado con contribuciones académicas, abordando aspectos de la literatura de retiros de productos en todas sus generalidades. El $46 \%$ presenta un enfoque del problema hacia lo práctico, es decir, hacia las diferentes implicaciones para preparar y desarrollar un retiro de productos. El $11 \%$ presenta un enfoque legal al problema, donde se abordan las diferentes acciones por parte de las agencias gubernamentales y legislaciones, como requisito previo a la responsabilidad de retiros de productos, bonos de reembolso, adquisición de nuevos productos, indemnizaciones, insolvencia económica y sanciones.

Según el tipo de metodología propuesta o aplicada, el $54 \%$ presenta un enfoque metodológico cualitativo, dado que se recopilan datos sobre las características, causas y consecuencias puntuales relacionadas con el retiro de productos, haciendo una descripción de la problemática sin adentrarse en temas como costos de campañas de retiro de productos, tratamiento de la problemática y/o cuantificación de un evento de recalls.

El $19 \%$ presenta un enfoque cuantitativo aplicando modelos matemáticos, números difusos, análisis financiero o econométrico y técnicas de estadística descriptiva, donde se comprueba la capacidad de los retiros de productos, consecuencias económicas y percepción de los consumidores tras el día del anuncio de un retiro de productos, en su mayoría, por medio de estudios de casos y aplicación de dichas metodologías.

El $23 \%$ presenta un enfoque mixto, donde se obtienen datos tanto descriptivos como predictivos, según las variables identificadas en el retiro de productos.

Según las características de los datos, el $60 \%$ utilizó datos reales para el análisis del problema, ejemplificando por medio de estudios de casos, entrevistas, encuestas, reportes, noticieros, bases de datos corporativas y procedentes de entes gubernamentales. El $49 \%$ utilizó datos sintéticos, a partir de la recolección de datos secundarios, recreando así otros casos de estudio y nuevas hipótesis. El $12 \%$ no consideró el uso de datos para el análisis de estudios de casos y se basó en los referentes teóricos.

En lo referente a la categoría del producto, el $32 \%$ de los artículos se refiere al análisis de retiros de productos en la industria de juguetes, el $35 \%$ abarca el sector automotor, el $14 \%$ aborda el sector de suministros eléctricos, el $12 \%$ analiza el sector de belleza y cuidado personal, el $9 \%$ aborda el análisis de la industria textil o vestimenta, el $37 \%$ analiza la industria de alimentos y sus efectos, el $20 \%$ analiza las implicaciones de la industria de medicamentos. En el $11 \%$ de los casos se analizan otro tipo de categoría varias. Finalmente, se resalta que en el $28 \%$ de los estudios se identifican aspectos del estado de la investigación, enfoques y metodologías relacionadas con el retiro de productos, proceso de producción o cadena de suministro sin tratar un sector específico.

\section{Conclusiones}

Los eventos de recalls son un problema importante de los mercados modernos, relacionados tanto con la gestión de la calidad como con la gestión de cadenas de suministro. A pesar de los grandes avances en los sistemas productivos y de control de la calidad, aún existe una brecha de conocimiento por resolver en la magnitud y frecuencia de los retiros de productos a nivel nacional e internacional. Esto se soporta en los hallazgos mostrados en este trabajo en lo referente al creciente número de eventos de recalls a nivel mundial y el desarrollo de iniciativas gubernamentales para enfrentar la problemática.

En este artículo se hizo un estudio de los aportes realizados en la literatura en los últimos 20 años para tratar el problema de los Recalls. 
Tabla 2. Clasificación taxonómica de estudios del problema de recalls

\begin{tabular}{|c|c|c|c|c|c|c|c|c|c|c|c|c|c|c|c|c|c|c|c|c|c|c|c|}
\hline \multirow[b]{2}{*}{ 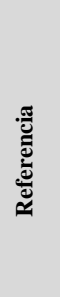 } & \multicolumn{2}{|c|}{$\begin{array}{l}\text { 1. Tipo } \\
\text { aporte }\end{array}$} & \multicolumn{3}{|c|}{$\begin{array}{l}\text { 2. Enfoque de } \\
\text { calidad }\end{array}$} & \multicolumn{3}{|c|}{$\begin{array}{l}\text { 3. Enfoque del } \\
\text { problema }\end{array}$} & \multicolumn{3}{|c|}{$\begin{array}{l}\text { 4. Tipo de } \\
\text { metodología }\end{array}$} & \multicolumn{3}{|c|}{$\begin{array}{l}\text { 5. Características } \\
\text { de los datos }\end{array}$} & \multicolumn{9}{|c|}{ 6. Categoría de producto } \\
\hline & 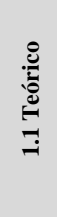 & 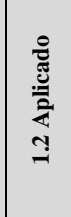 & 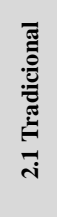 & 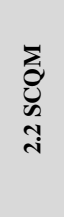 & 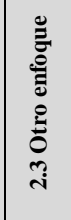 & 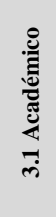 & 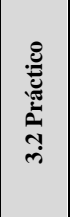 & 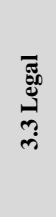 & 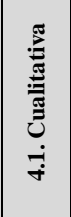 & 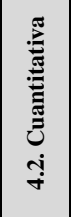 & 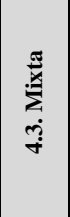 & 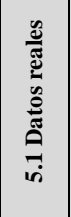 & 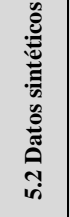 & 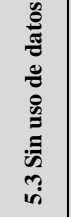 & 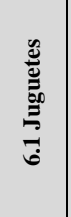 & 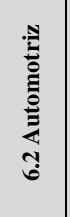 & 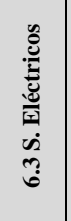 & 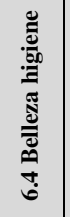 & 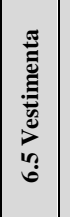 & 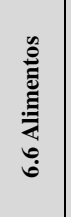 & 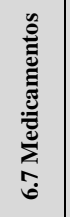 & 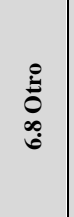 & 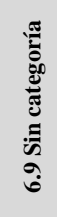 \\
\hline$[1]$ & $\mathrm{X}$ & & $\mathrm{X}$ & & & $\mathrm{X}$ & & & & & $\mathrm{X}$ & $\mathrm{X}$ & & & & $\mathrm{X}$ & & & & & $\mathrm{X}$ & & \\
\hline [2] & $\mathrm{x}$ & & & & $\mathrm{x}$ & $\mathrm{X}$ & & $\mathrm{x}$ & & & & $\mathrm{x}$ & & & & & & & & & & & $\mathrm{x}$ \\
\hline$[3]$ & $\mathrm{x}$ & & & $\mathrm{x}$ & & $\mathrm{x}$ & & & $\mathrm{X}$ & & & & $\mathrm{x}$ & & & $\mathrm{x}$ & & & & & & & \\
\hline [4] & $\mathrm{X}$ & & $\mathrm{X}$ & & & $\mathrm{X}$ & & & $\mathrm{X}$ & & & & $\mathrm{X}$ & & & & & & & & & & $\mathrm{X}$ \\
\hline [5] & & $\mathrm{X}$ & & $\mathrm{X}$ & & & $\mathrm{X}$ & & & $\mathrm{x}$ & & & $\mathrm{x}$ & & & & & & & & & & $\mathrm{x}$ \\
\hline [9] & $\mathrm{X}$ & & & $\mathrm{X}$ & & $\mathrm{X}$ & & & $\mathrm{X}$ & & & $\mathrm{x}$ & & & $\mathrm{x}$ & & & & & & & & \\
\hline [10] & & $\mathrm{X}$ & & & $\mathrm{x}$ & & $\mathrm{X}$ & & & & $\mathrm{X}$ & $\mathrm{X}$ & $\mathrm{X}$ & & $\mathrm{X}$ & $\mathrm{x}$ & $\mathrm{X}$ & $\mathrm{X}$ & $\mathrm{X}$ & $\mathrm{X}$ & & $\mathrm{X}$ & \\
\hline [11] & $\mathrm{X}$ & & & $\mathrm{X}$ & & $\mathrm{X}$ & & & $\mathrm{X}$ & & & & $\mathrm{X}$ & & & & & & & & & & $\mathrm{X}$ \\
\hline [12] & $\mathrm{X}$ & & & $\mathrm{X}$ & & $\mathrm{X}$ & & & $\mathrm{X}$ & & & $\mathrm{X}$ & & & $\mathrm{X}$ & $\mathrm{X}$ & & $\mathrm{X}$ & & $\mathrm{X}$ & & & \\
\hline [13] & $\mathrm{x}$ & & & $\mathrm{X}$ & & $\mathrm{x}$ & & & $\mathrm{x}$ & & & & $\mathrm{X}$ & & & & & & & & & & $\mathrm{X}$ \\
\hline$[14]$ & $\frac{\alpha}{x}$ & & & $\frac{\mathrm{x}}{\mathrm{X}}$ & & $\frac{x}{x}$ & & & & & & $\mathrm{X}$ & & & & $\mathrm{x}$ & & & & & & & \\
\hline$[16]$ & & $\mathrm{X}$ & & $\mathrm{X}$ & & & $\mathrm{X}$ & & & & $\mathrm{X}$ & & $\mathrm{X}$ & & & $\mathrm{x}$ & & & & & & & \\
\hline$[17]$ & & $\mathrm{x}$ & & & $\mathrm{X}$ & & $\mathrm{x}$ & & & & $\mathrm{x}$ & $\mathrm{X}$ & $\mathrm{x}$ & & & & & & & & & & $\mathrm{X}$ \\
\hline$[18]$ & & $\mathrm{x}$ & & & $\mathrm{X}$ & & $\mathrm{x}$ & & & $\mathrm{X}$ & & $\mathrm{x}$ & $\mathrm{X}$ & & & & & & & $\mathrm{X}$ & & & \\
\hline [19] & $\mathrm{X}$ & & & $\mathrm{X}$ & & $\mathrm{X}$ & & & $\mathrm{X}$ & & & & & $\mathrm{x}$ & & & & & & & & & \\
\hline [20] & $\mathrm{x}$ & & $\mathrm{X}$ & $\mathrm{X}$ & & $\mathrm{X}$ & $\mathrm{X}$ & & $\mathrm{x}$ & & & $\mathrm{x}$ & & & $\mathrm{x}$ & & & & & & & & \\
\hline [21] & $\mathrm{X}$ & & & $\mathrm{X}$ & & $\mathrm{X}$ & & & $\mathrm{X}$ & & & & & $\mathrm{X}$ & $\mathrm{X}$ & $\mathrm{X}$ & & & & & & & \\
\hline [22] & $\mathrm{X}$ & $\mathrm{X}$ & $\mathrm{X}$ & & & $\mathrm{X}$ & & & & & $\mathrm{X}$ & $\mathrm{X}$ & $\mathrm{x}$ & & $\mathrm{X}$ & & & & & & & & \\
\hline$[23]$ & & $\mathrm{X}$ & & & $X$ & & $\mathrm{X}$ & & & & $\mathrm{X}$ & $\mathrm{X}$ & $\mathrm{X}$ & & $X$ & & & & & & & & \\
\hline$[24]$ & & $\mathrm{X}$ & & $\mathrm{X}$ & & & $x$ & & & & $\mathrm{x}$ & & $\mathrm{X}$ & & & & & & & $\mathrm{X}$ & & & \\
\hline$[25]$ & $\mathrm{X}$ & & $X$ & $\mathrm{X}$ & & & $\mathrm{X}$ & & $\mathrm{X}$ & & & $\mathrm{X}$ & & & & & & & & & & & $\mathrm{X}$ \\
\hline$[26]$ & $\mathrm{X}$ & & $\mathrm{X}$ & $\begin{array}{l}x \\
\end{array}$ & & $\mathrm{X}$ & & & $\mathrm{X}$ & & & $\mathrm{X}$ & & & $\mathrm{X}$ & $\mathrm{X}$ & & & & & & & \\
\hline$[27]$ & $x$ & & & $\mathrm{X}$ & & & $\mathrm{X}$ & & & $X$ & & & $X$ & & $\mathrm{X}$ & & & & & & & & \\
\hline$[28]$ & $\frac{x}{x}$ & & $\mathrm{X}$ & 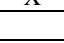 & & $\mathrm{X}$ & $\Lambda$ & & $\mathrm{X}$ & & & & & $\mathrm{X}$ & & & & & & & & & $\mathrm{X}$ \\
\hline$[29]$ & $X$ & & & $X$ & & $X$ & & & $\mathrm{X}$ & & & & & $X$ & & & & & & & & & $x$ \\
\hline$[30]$ & & $\mathrm{X}$ & & $x$ & & & $X$ & & & $x$ & & & $\mathrm{X}$ & & & & & & & $\mathrm{X}$ & & & \\
\hline$[31]$ & & $x$ & & & 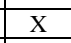 & & $x$ & & & $x$ & & & $\mathrm{X}$ & & & & & & & & & & \\
\hline$[32]$ & $X$ & & & $\mathrm{X}$ & & $\mathrm{X}$ & $\mathrm{X}$ & $x$ & & & $\mathrm{X}$ & $\mathrm{X}$ & $\mathrm{X}$ & & $\mathrm{X}$ & $\mathrm{X}$ & & $\mathrm{X}$ & & $\mathrm{X}$ & & & \\
\hline$[33]$ & $x$ & & & & $X$ & $x$ & & & $X$ & & & & $x$ & & & & & & & $x$ & & & 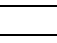 \\
\hline$[34]$ & $x$ & & $X$ & & & $x$ & & $\mathrm{X}$ & $x$ & & & $\mathrm{X}$ & & & & & & & & $\mathrm{X}$ & & & \\
\hline$[35]$ & & $\mathrm{X}$ & & $\mathrm{X}$ & & & $\mathrm{X}$ & & & $x$ & & & $\mathrm{X}$ & & & & & & & & & & $\mathrm{X}$ \\
\hline [36] & & $\mathrm{x}$ & & $\mathrm{x}$ & & & $\mathrm{x}$ & & & $x$ & & & $x$ & & $\mathrm{X}$ & $\mathrm{X}$ & & $\mathrm{X}$ & & $\mathrm{X}$ & $\mathrm{X}$ & & $\Lambda$ \\
\hline [37] & & $\mathrm{x}$ & $\mathrm{X}$ & & & & $\mathrm{X}$ & & & $\mathrm{X}$ & & $\mathrm{X}$ & $\mathrm{X}$ & & & $\mathrm{X}$ & $x$ & & & $\mathrm{X}$ & $X$ & & \\
\hline$[38]$ & $x$ & & & $\mathrm{X}$ & & $\mathrm{X}$ & $\mathrm{X}$ & 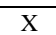 & $\mathrm{X}$ & & & $\mathrm{X}$ & & & & & & & & & $\mathrm{X}$ & & \\
\hline [39] & & $\mathrm{X}$ & & $\mathrm{X}$ & & & $\mathrm{X}$ & & $\mathrm{X}$ & & & $\mathrm{X}$ & $\mathrm{X}$ & & & & & & & $\mathrm{X}$ & & & \\
\hline$[40]$ & & $X$ & & $X$ & & & $\mathrm{X}$ & & $X$ & & & $\mathrm{X}$ & $\mathrm{X}$ & & $\mathrm{X}$ & $\mathrm{X}$ & $\mathrm{X}$ & & & & $\mathrm{X}$ & & \\
\hline [41] & $x$ & & & $\mathrm{X}$ & & $\mathrm{X}$ & & & $\mathrm{X}$ & & & & & $\mathrm{X}$ & & & & & & & & & $\mathrm{X}$ \\
\hline [42] & $x$ & & & $\mathrm{X}$ & & & $X$ & & $\mathrm{X}$ & & & $\mathrm{X}$ & $X$ & & $x$ & & & & & $\mathrm{X}$ & & & \\
\hline [43] & $x$ & & & & $\mathrm{X}$ & & $\begin{array}{l}x \\
x\end{array}$ & & & $\mathrm{X}$ & & $x$ & $\frac{\alpha}{x}$ & & & $\mathrm{X}$ & & & & & & $\mathrm{X}$ & \\
\hline$[44]$ & $X$ & & & $X$ & & $X$ & & & $X$ & & & $\mathrm{X}$ & & & $\mathrm{X}$ & $\mathrm{X}$ & $\mathrm{X}$ & & $X$ & $\mathrm{X}$ & $\mathrm{X}$ & & \\
\hline [45] & & $\mathrm{X}$ & & $X$ & & & $\mathrm{X}$ & & & $\mathrm{X}$ & & & $\mathrm{X}$ & & & & & & & & & & $\mathrm{X}$ \\
\hline [46] & $X$ & & & $x$ & & $\mathrm{X}$ & & & $\mathrm{X}$ & & & & & $x$ & & & & & & & & & $x$ \\
\hline [47] & $\mathrm{X}$ & & & $\mathrm{X}$ & & $\mathrm{X}$ & & & & & $\mathrm{X}$ & $\mathrm{X}$ & & & & & & & & $\mathrm{X}$ & & $\mathrm{X}$ & \\
\hline$[48]$ & $\mathrm{X}$ & & & $\mathrm{X}$ & & $\mathrm{X}$ & & & $\mathrm{X}$ & & & & & $\mathrm{X}$ & & & & & & & & & $\mathrm{X}$ \\
\hline [49] & $\mathrm{X}$ & & & & $\mathrm{X}$ & $\mathrm{X}$ & & & $\mathrm{X}$ & & & $\mathrm{X}$ & & & & & & & & $\mathrm{X}$ & & & \\
\hline [50] & $\mathrm{X}$ & & & & $\mathrm{X}$ & & $\mathrm{X}$ & & & & $\mathrm{X}$ & $\mathrm{X}$ & $\mathrm{X}$ & & $\mathrm{X}$ & $\mathrm{X}$ & $X$ & $X$ & $\mathrm{X}$ & $\mathrm{X}$ & $\mathrm{x}$ & $\mathrm{X}$ & \\
\hline [51] & $\mathrm{X}$ & & & & $x$ & & $\alpha$ & $\mathrm{x}$ & $\mathrm{X}$ & & & $\mathrm{X}$ & & & $x$ & $\mathrm{X}$ & $\mathrm{X}$ & $\mathrm{X}$ & $\mathrm{X}$ & $\mathrm{x}$ & $x$ & $x$ & \\
\hline [52] & $\mathrm{X}$ & & & & $X$ & & $\mathrm{X}$ & & & $X$ & & $\mathrm{X}$ & & & $X$ & $\mathrm{X}$ & $\mathrm{X}$ & $X$ & $X$ & $\mathrm{X}$ & $X$ & $\mathrm{X}$ & \\
\hline$[53]$ & $X$ & & $\mathrm{X}$ & & & $X$ & & $X$ & & & $X$ & $\mathrm{X}$ & & & & & & & & $\mathrm{X}$ & & & \\
\hline [54] & $x$ & & $\alpha$ & $\mathrm{X}$ & & $\mathrm{X}$ & & & $\mathrm{X}$ & & & $x$ & & & & & & & & & & & $\mathrm{X}$ \\
\hline$[55]$ & 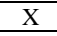 & & & & $\mathrm{X}$ & $\begin{array}{l}X \\
\end{array}$ & & & $\mathrm{X}$ & & & $\mathrm{X}$ & & & & & $\mathrm{X}$ & & & & & & \\
\hline$[56]$ & $\mathrm{X}$ & & $\mathrm{X}$ & & & $\mathrm{X}$ & & & & & $\mathrm{X}$ & $\mathrm{X}$ & & & & $\mathrm{X}$ & & & & & & & \\
\hline$[57]$ & $x$ & & & & $\mathrm{X}$ & $\mathrm{X}$ & & & & & $\mathrm{X}$ & $x$ & & & & & & & & $\mathrm{X}$ & & & \\
\hline$[58]$ & $x$ & & & $\mathrm{X}$ & & $x$ & & & $\mathrm{X}$ & & & $x$ & & & & & & & & & & & $x$ \\
\hline [59] & $x$ & & & $\mathrm{X}$ & & $\mathrm{X}$ & & & $x$ & & & $\mathrm{X}$ & & & & & & & & & $X$ & & \\
\hline$[60]$ & $\mathrm{X}$ & & & $\mathrm{X}$ & & & $\mathrm{X}$ & & $\mathrm{x}$ & & & & $\mathrm{X}$ & & & $\mathrm{X}$ & & & & $\mathrm{X}$ & $X$ & & \\
\hline$[61]$ & $\mathrm{X}$ & & & $\mathrm{X}$ & & & $\mathrm{X}$ & & $\mathrm{X}$ & & & & $\mathrm{x}$ & & $\mathrm{X}$ & $\mathrm{X}$ & & & & & $\mathrm{X}$ & & \\
\hline Total & $74 \%$ & $28 \%$ & $19 \%$ & $61 \%$ & $25 \%$ & $58 \%$ & $46 \%$ & $11 \%$ & $54 \%$ & $\mid 19 \%$ & $23 \%$ & \begin{tabular}{|l|l|}
$60 \%$ \\
\end{tabular} & $49 \%$ & $12 \%$ & $32 \%$ & $35 \%$ & $14 \%$ & $12 \%$ & $9 \%$ & $37 \%$ & $21 \%$ & $11 \%$ & $28 \%$ \\
\hline
\end{tabular}

Fuente: elaboración propia. 
Para ello, se realizó un análisis bibliométrico y una clasificación taxonómica posterior, la cual permitió identificar los diferentes enfoques, metodologías y características de los trabajos realizados.

Por el contrario, los países en vías de desarrollo Si bien la mayoría de los aportes tiene un enfoque esencialmente teórico-académico, también se han desarrollado estudios de casos en los que se analizan las principales categorías de mayor predominancia como lo son la industria de juguetes, el sector automotor, la industria de suministros eléctricos y la industria alimenticia.

La propuesta de clasificación taxonómica desarrollada en este artículo constituye un enfoque novedoso para analizar los estudios previos sobre recalls, y constituye un punto de partida para el desarrollo de futuras investigaciones. La taxonomía desarrollada se puede ampliar a medida que aumente el número de contribuciones académicas al estudio de los recalls. Este artículo es producto de una investigación en curso sobre el modelado de la calidad en cadenas de suministro. Los trabajos futuros se centrarán en desarrollar modelos matemáticos que incorporen los riesgos e incertidumbres propios de las cadenas de suministro y permitan cuantificar el impacto de los recalls sobre el desempeño global.

\section{Referencias}

[1] D. C. Gibson, "Public relations considerations of consumer product recall", Public Relat. Rev., vol. 21, no. 3 , pp. $225-240, \quad 1995$, doi: 10.1016/03638111(95)90023-3

[2] T. Copeland, G. Jackson, F. Morgan, "An Update on Product Recalls", J. Mark. Channels, vol. 11, no. 2-3, pp. 103-121, 2004, doi: 10.1300/j049v11n02_06

[3] E. C. Baraldi y P. C. Kaminski, "A study on the causes of recall in automotive vehicles marketed in Brazil", en $25^{\circ}$ Congreso y Exhibición Internacional SAE BRASIL, 2016, pp. 128-133, doi: 10.4271/2016-36-0169

[4] A. Gunasekaran, N. Subramanian, W.T.E. Ngai, "Quality management in the 21st century enterprises: Research pathway towards Industry 4.0", Int. J. Prod. Econ., vol. 207, pp. 125-129, 2019, doi: 10.1016/j.ijpe.2018.09.005

[5] K. R. Balachandran, S. Radhakrishnan, "Quality implications of warranties in a supply chain", Manage. Sci., vol. 51, no. 8, pp. 1266-1277, 2005, doi: $10.1287 /$ mnsc. 1050.0408
[6] L. A. Saavedra-Robinson, S. Páez-Sarmiento, J. F. Ramírez, "Human error assessment in supply chain: case study in road transport services", Rev. UIS Ing., vol. 19, no. 4 , pp. 287-300, 2020, doi: 10.18273/revuin.v19n42020024

[7] Y. Torres-Medina, "The analysis of human error in manufacturing: a key to improve production quality", Rev. UIS Ing., vol. 19, no. 4, pp. 53-62, 2020, doi: 10.18273/revuin.v19n4-2020005

[8] T. J. Fontalvo Herrera, J. Morelos Gómez, y A. Mendoza Mendoza, "Impact of ISO 9001 certification in productivity and profitability indicators in companies of Zone Franca- Barranquilla through discriminant analisys", Rev. UIS Ing., vol. 11, no. 2, pp. 215-225, 2012.

[9] L. C. Wood, J. X. Wang, K. Olesen, T. Reiners, "The effect of slack, diversification, and time to recall on stock market reaction to toy recalls", Int. J. Prod. Econ., vol. 193, pp. 244-258, 2017, doi: 10.1016/j.ijpe.2017.07.021

[10] T. H. Chu, C. C. Lin, L. J. Prather, "An extension of security price reactions around product recall announcements", Q. J. Bus. Econ., vol. 44, no. 3/4, pp. 33-48, 2005, doi: $10.2307 / 40473403$

[11] H. Zhen, L. Ckm, K. L. Choy, "A quality risk model based on supply chain perspective", en SCMIS 2010 Proc. 2010 8th Int. Conf. Supply Chain Manag. Inf. Syst. Logist. Syst. Eng., 2010, pp. 1-6.

[12] G. Grabowski, J. L. Hertzberg, "Avoiding and managing product recalls", Risk Management Magazine, vol. 54, no. 12, pp. 12-17, 2007.

[13] G. Liotta, A. Chaudhuri, "Minimizing recall risk by collaborative digitized information sharing between OEM and suppliers: A simulation based investigation", en Proc. - Winter Simul. Conf., 2017, pp. 2454-2465.

[14] R. L. Bray, J. C. Serpa, A. Colak, "Supply chain proximity and product quality", Manage. Sci., vol. 65, no. 9, pp. 4079-4099, 2019, doi: $10.1287 /$ mnsc. 2018.3161

[15] A. Reisman, "How can or/ms educators benefit from creating and using taxonomies?", Informs, vol. 4, no. 3, pp. 55-65, 2004, doi: 10.1287/ited.4.3.55

[16] J. Fan, D. Ni, X. Fang, "Liability cost sharing, product quality choice, and coordination in two-echelon supply chains", Eur. J. Oper. Res., vol. 284, no. 2, pp. 514-537, 2020, doi: 10.1016/j.ejor.2020.01.003 
[17] C. A. De Matos, C. A. Vargas Rossi, "Consumer reaction to product recalls: factors influencing product judgement and behavioural intentions", Int. J. Consum. Stud., vol. 31, pp. 109-116, 2007, doi: 10.1111/j.14706431.2006.00499.x

[18] H. Van Heerde, K. Helsen, M. G. Dekimpe, "The impact of a product-harm crisis on marketing effectiveness", Mark. Sci., vol. 26, no. 2, pp. 230-245, 2007, doi: $10.1287 / \mathrm{mksc} .1060 .0227$

[19] M. A. Lyles, B. B. Flynn, M. T. Frohlich, "All supply chains don't flow through: understanding supply chain issues in product recalls", Manag. Organ. Rev., vol. 4, no. 2, pp. 167-182, 2008, doi: 10.1111/j.17408784.2008.00106.x

[20] C. S. Tang, "Making products safe: process and challenges", International Commerce Review, vol. 8, no. 1, pp. 48-55, 2008, doi: 10.1007/s12146-008-0028-2

[21] M. Etayankara, H. Bapuji, "Product recalls: a review of literature", en Proceedings of the Annual Conference of ASAC, Niagara Falls, Ontario, 2009, pp. 44-59.

[22] H. K. Jung, "Product recalls: consumer reaction and blame attributions", tesis doctoral, The University of Wisconsin, 2009.

[23] Y. Chen, S. Ganesan, Y. Liu, "Does a firm's product-recall strategy affect its financial value? an examination of strategic alternatives during productharm crises", J. Mark., vol. 73, pp. 214-226, 2009, doi: 10.1509/jmkg.73.6.214

[24] A. G. J. Velthuis, M. W. Reij, K. Baritakis, M. Dang, C. P. A. Wagenberg, "Recall costs balanced against spoilage control in dutch custard", J. Dairy Sci., vol. 93, no. 6, pp. 2779-2791, 2010, doi: 10.3168/jds.2009-2654

[25] D. Pyke, C. S. Tang, "How to mitigate product safety risks proactively? process, challenges and opportunities", Int. J. Logist. Res. Appl., vol. 13, no. 4, pp. 243-256, 2010, doi: 10.1080/13675561003720214

[26] S. Kumar, S. Schmitz, "Managing recalls in a consumer product supply chain - root cause analysis and measures to mitigate risks", Int. J. Prod. Res., vol. 49, no. 1, pp. 235-253, 2011, doi: $10.1080 / 00207543.2010 .508952$

[27] Y. K. Tse, K. H. Tan, "Managing product quality risk in a multi-tier global supply chain", Int. J. Prod. Res., vol. 49, no. 1, pp. 139-158, 2011, doi: 10.1080/00207543.2010.508942
[28] D. Lei, S. Xiao, "Building the information management system of defective product recalls", en 2011 IEEE 3rd International Conference on Communication Software and Networks, ICCSN 2011, 2011, pp. 372-375, doi: 10.1109/ICCSN.2011.6014290

[29] L. Li, J. N. Warfield, "Perspectives on quality coordination and assurance in global supply chains", Int. J. Prod. Res., vol. 49, no. 1, pp. 1-4, 2011, doi: 10.1080/00207543.2010.508932

[30] X. Wang, D. Li, X. Shi, “A fuzzy model for aggregative food safety risk assessment in food supply chains", Prod. Plan. Control, vol. 23, no. 5, pp. 377-395, 2012, doi: 10.1080/09537287.2011.561812

[31] F. Magno, "Managing product recalls: The effects of time, responsible vs. opportunistic recall management and blame on consumers' attitudes", Procedia-Soc. Behav. Sci., vol. 58, pp. 1309-1315, 2012, doi: 10.1016/j.sbspro.2012.09.1114

[32] B. B. Flynn, X. Zhao, Global supply chain quality management: Product recalls and their impact, 1 st ed. Boca Raton, FL, USA: CRC Press, 2012.

[33] O. Thompson, "Recall prevention and planning", 2012. [En línea]. Disponible en: https://www.foodengineeringmag.com/articles/89674recall-prevention-and-planning.

[34] A. Potter, J. Murray, B. Lawson, S. Graham, "Trends in product recalls within the agri-food industry: empirical evidence from the USA, UK and the Republic of Ireland", Trends Food Sci. Technol., vol. 28, pp. 7786, 2012, doi: 10.1016/j.tifs.2012.06.017

[35] Y. K. Tse, K. H. Tan, "Managing product quality risk and visibility in multi-layer supply chain", Int. J. Prod. Econ., vol. 139, pp. 49-57, 2012, doi: 10.1016/j.ijpe.2011.10.031

[36] H. Haiju, "Three essays on supply chain quality management and product recalls", tesis doctoral, The Chinese University of Hong Kong, 2013.

[37] X. Zhao, Y. Li, B. B. Flynn, "The financial impact of product recall announcements in China", Int. J. Prod. Econ., vol. 142, pp. 115-123, 2013, doi: 10.1016/j.ijpe.2012.10.018

[38] D. Raheja, Preventing Medical Device Recalls. Boca Raton, FL, USA: CRC Press, 2014. 
[39] S. Kumar, "A knowledge based reliability engineering approach to manage product safety and recalls", Expert Syst. Appl., vol. 41, pp. 5323-5339, 2014, doi: 10.1016/j.eswa.2014.03.007

[40] A. Sharma, D. Garg, A. Agarwal, "Product recall: supply chain quality issue?", Int. J. Intell. Enterp., vol. 2, no. 4, pp. 277-293, 2014, doi: 10.1504/IJIE.2014.069059

[41] D. J. Ketchen, K. D. Wowak, C. W. Craighead, "Resource gaps and resource orchestration shortfalls in supply chain management: the case of product recalls", J. Supply Chain Manag., vol. 50, no. 3, pp. 6-15, 2014, doi: $10.1111 /$ jscm. 12047

[42] J. Z. Ni, B. B. Flynn, F. R. Jacobs, "Impact of product recall announcements on retailers' financial value", Int. J. Prod. Econ., vol. 153, pp. 309-322, 2014, doi: 10.1016/j.ijpe.2014.03.014

[43] F. Germann, R. Grewal, W. T. Ross Jr, R. K. Srivastava, "Product recalls and the moderating role of brand commitment", Mark. Lett., vol. 25, pp. 179-191, 2014, doi: 10.1007/s11002-013-9250-5

[44] K. Ahsan, I. Gunawan, "Analysis of product recalls: identification of recall initiators and causes of recall", Oper. Supply Chain Manag., vol. 7, no. 3, pp. 97-106, 2014, doi: 10.31387/oscm0180115

[45] A. B. Steven, Y. Dong, T. Corsi, "Global sourcing and quality recalls: an empirical study of outsourcingsupplier concentration-product recalls linkages", J. Oper. Manag., vol. 32, pp. 241-253, 2014, doi: 10.1016/j.jom.2014.04.003

[46] K. D. Wowak, C. A. Boone, "So many recalls, so little research: a review of the literature and road map for future research", J. Supply Chain Manag., vol. 51, no. 4, pp. 54-72, 2015, doi: 10.1111/jscm. 12079

[47] J. Crumbly, L. Carter, "The impact of information technology on product recalls: exploring the role of the six 'Ts' of supply chain management", Prod. Plan. Control, vol. 26, no. 12, pp. 958-968, 2015, doi: 10.1080/09537287.2015.1009521

[48] A. B. Steven, "Supply chain structure, product recalls, and firm performance: empirically investigating recall drivers and recall financial performance relationships", Decis. Sci., vol. 46, no. 2, pp. 477-483, 2015, doi: 10.1111/deci. 12135
[49] J. J. Bejarano-Roncancio, A. C. Díaz-Moreno, M. J. Egoavil-Cardozo, "Recall en la industria alimentaria: una estrategia sanitaria por implementar en Colombia", Rev. la Fac. Med., vol. 64, no. 4, pp. 727-734, 2016, doi: 10.15446/revfacmed.v64n4.52915

[50] L. Hsu, B. Lawrence, "The role of social media and brand equity during a product recall crisis: a shareholder value perspective", Int. J. Res. Mark., vol. 33, pp. 59-77, 2016, doi: 10.1016/j.ijresmar.2015.04.004

[51] J. Wieber Lens, "Product recalls: why is tort law deferring to agency inaction?", St. Johns. Law Review., vol. 90, no. 329, pp. 329-367, 2016.

[52] O. Kini, J. Shenoy, V. Subramaniam, "Impact of financial leverage on the incidence and severity of product failures: evidence from product recalls", Rev. Financ. Stud., vol. 30, no. 5, pp. 1790-1829, 2017, doi: 10.1093/rfs/hhw092

[53] T. D. Johnson-Hall, "Ensuring Food Safety by Preventing Food Recalls: The Impact of Locus of Failure, Regulatory Agency Discovery, Breadth, and Firm Size on Corrective Action", J. Mark. Channels, vol. 24, no. 34, pp. 115-135, 2017, doi: 10.1080/1046669X.2017.1393230

[54] R. Sreedevi, H. Saranga, "Uncertainty and supply chain risk: the moderating role of supply chain flexibility in risk mitigation", Int. J. Prod. Econ., vol. 193, pp. 332342, 2017, doi: 10.1016/j.ijpe.2017.07.024

[55] O. Unsal, M. K. Hassan, D. Zirek, "Product recalls and security prices: new evidence from the US market", J. Econ. Bus., vol. 93, pp. 62-79, 2017, doi: 10.1016/j.jeconbus.2017.07.003

[56] O. Topaloglu, O. N. Gokalp, "How brand concept affects consumer response to product recalls: a longitudinal study in the U.S. auto industry", J. Bus. Res., vol. 88, pp. 245-254, 2018, doi: 10.1016/j.jbusres.2018.03.035

[57] D. Kong, L. Shi, Z. Yang, "Product recalls, corporate social responsibility, and firm value: evidence from the Chinese food industry", Food Policy., vol. 83, pp. 60-69, 2019, doi: 10.1016/j.foodpol.2018.11.005

[58] A. T. Mayounga, "Antecedents of recalls prevention : analysis and synthesis of research on product recalls", Supply Chain Forum An Int. J., vol. 19, no. 3, pp. 164-177, 2018, doi: $10.1080 / 16258312.2018 .1530575$ 
[59] M. Bruccoleri, G. Perrone, E. Mazzola, R. Handfield, "The magnitude of a product recall: offshore outsourcing vs. captive offshoring effects", Int. J. Prod. Res., vol. 57, no. 13, pp. 1-17, 2018, doi: $10.1080 / 00207543.2018 .1533652$

[60] M. Zhang, H. Hu, X. Zhao, "Developing product recall capability through supply chain quality management", Int. J. Prod. Econ., vol. 229, pp. 1-2, 2020, doi: 10.1016/j.ijpe.2020.107795

[61] B. Dai, Y. Nu, X. Xie, J. Li, "Interactions of traceability and reliability optimization in a competitive supply chain with product recall", Eur. J. Oper. Res., vol. 290, no. 1, pp. 116-131, 2021, doi: 10.1016/j.ejor.2020.08.003 